\title{
ARTIFICIAL INTELLIGENCE FOR THE PREDICTION OF BLADDER CANCER
}

\author{
M.F. ABbod', J.W.F. CatTo ${ }^{2}$, M. ChEN' ${ }^{\prime}$, D.A. LINKENS', F.C. HAMDY ${ }^{2}$ \\ 'Department of Automatic Control and Systems Engineering, \\ ${ }^{2}$ The Academic Urology Unit, University of Sheffield. Sheffield. \\ United Kingdom
}

\begin{abstract}
New techniques for the prediction of tumour behaviour are needed as statistical analysis has a poor accuracy and is not applicable to the individual. Artificial intelligence (AI) may provide these suitable methods. We have previously shown that the predictive accuracies of neuro-fuzzy modelling (NFM) and artificial neural networks (ANN), two methods of AI, are superior to traditional statistical methods for the behaviour of bladder cancer (Catto et al, 2003). In this paper, we explain the Al techniques required to produce these predictive models. We used 9 parameters, which were a combination of experimental molecular biomarkers and conventional clinicopathological data, to predict the risk of tumour progression in a population of 109 patients with bladder cancer. NFM, using fuzzy logic to model data, achieved similar or superior predictive accuracy to ANN, which required cross-validation. However, unlike the impenetrable opaque structure of neural networks, the rules of NFM are transparent, enabling validation from clinical knowledge and the manipulation of input variables to allow exploratory predictions.
\end{abstract}

Biomed Eng Appl Basis Comm, 2004(April); 16: 49-58.

Keywords: Artificial Neural Network, Neuro-Fuzzy Modelling, Bladder Cancer, Prognostic prediction, $\mathrm{p} 53$, Mismatch repair.

\section{INTRODUCTION}

Cancer is a common disease, accounting for $25 \%$ of all deaths in the United Kingdom. Like many forms of malignancy, bladder cancer, the 4th commonest cancer amongst men, is increasing in incidence (by 5 $20 \%$ every 5 years (Quinn, 2000)). In 1994, over 11,000 new cases and over 5000 deaths due to transitional cell carcinoma of the bladder (TCC) were recorded (Quinn, 2000). At presentation, 70\% of TCCs

Received: Dec 23, 2003; Accepted: Jan 29, 2004

Correspondence: M.F. Abbod

Department of Automatic Control and Systems Engineering, University of Sheffield, Sheffield.

United Kingdom

E-mail: m.f.abbod@sheffield.ac.uk (the most common pathological variety) are superficial and non-invasive. They can be managed safely by a combination of local endoscopic resection and intravesical chemotherapy. Following treatment, these tumours require regular cystoscopic surveillance (Hall, et al, 1994) as $50 \%$ will recur as similar non-invasive lesions, and a smaller percentage $(20 \%)$ will progress to muscle invasion. Muscle invasive tumours have a poor prognosis ( $50 \% 5$ year survival rates) and require radical therapy if cure is to be achieved (Stein et al, 2001). Following radical treatment, adjuvant chemotherapy can be used to reduce relapse and possibly mortality rates (Raghaven et al, 2002). However, due to the toxicity of chemotherapy and the current literature evidence of benefit, it is used most frequently in those cases with pathologically confimed nodal metastasis (i.e. at high relapse risk).

An accurate prediction of the behaviour of an 
individual's cancer would benefit both the patient and the physician. Patients with non-relapsing tumours could be safely reassured and discharged, resulting in both financial and psychological benefits, whilst those relapsing tumours could be monitored more frequently and treated more aggressively. Currently, the pathological stage and grade of a cancer are the most reliable predictors of tumour behaviour (TNM classification) (Sobin and Wittekind, 1997). In addition, for some specific tumours there are further prognostic factors. For example, in superficial TCC the presence of carcinoma in situ and the state of the bladder 3 months after surgery are additional important prognostic factors (Parmar et al, 1989), whilst in muscle invasive TCC the state of the resected lymph nodes gives the most additional information (Stein et al, 2001). Although these parameters are useful at stratifying patients into subgroups of relapse risk, it is still not easy to predict the behaviour of individual tumours.

It is believed that the phenotype of a tumour reflects it genotype. Thus, the number and type of molecular alteration (gene deletions, mutations etc.) found in a cell, dictate its pathogenicity. Using this knowledge, it seems likely that these molecular changes can be used to predict a cell's behaviour. Some of the most biologically promising 'biomarkers' are the p53 and mismatch repair proteins (MMR) hMSH2 and hMLH1. The p53 gene is estimated to be mutated in over $50 \%$ of human cancers (Hollstein et al, 1991) and has been shown to predict recurrence and survival in bladder cancer (Esrig et al, 1994) of the MMR proteins (most frequently hMSH2 and hMLH1) results in microsatellite instability and subsequent carcinogenesis in hereditary (Aaltonen et al, 1993) and sporadic colorectal cancer (Liu et al, 1995). The resultant tumours have a distinctive phenotype with chemoresistance (Drummond et al, 1996) and better than expected (for their stage) clinical outcomes (Gryfe et al, 2000). However, despite optimistic reports for these and other molecular biomarkers, to date no single molecule is sufficiently robust to use in routine clinical practice.

An alternative solution to the problem of predicting tumour behaviour accurately, lies potentially within the interpretation of data (Catto et al, 2003). Traditional statistical methods, e.g. logistic regression, produce probabilities of behaviour that may be generalisable to a population but not predictive for an individual. Never the less, a predictive accuracy of up to $70 \%$ can be achieved for tumours using the TNM classification (Burke et al, 1997). On the other hand, using artificial intelligence (AI) methodologies, such as artificial neural networks (ANN) and neuro-fuzzy modelling (NFM), complex non-linear relationships between dependant and independent variables, within a population whose distribution may not be normal, can be identified.

Multi-Layer Perceptron ANNs have been applied to clinical medicine since 1989 (Baxt, 1989). Previous authors have shown that ANNs are superior to standard statistical analysis in the diagnosis of chest pain (Baxt, 1995), the TNM staging system at predicting breast and colorectal cancer outcomes 14 and predicting progression of poorly differentiated superficial bladder tumours (G3pT1) (Qureshi et al, 2000). However, ANNs have some problems such as overtraining which incorporate the inherent variation 'noise' of a sample population. An alternative solution ids to use NFM technique which simple and efective method for selecting significant input variables and determining optimal number of fuzzy rules when building a model from data. The NFM approach, both the input selection and partition validating are determined on the basis of a class of sub-clusters created by a self-organising network. The important input variables which independently and significantly influence the system output can be extracted by a fuzzy neural network. On the other hand, the optimal number of fuzzy rules can be determined separately via the fuzzy c-means algorithm with a modified fuzzy entropy as the criterion of cluster validation.

We have recently compared the predictive accuracies of NFM and ANN with traditional statistical methods, in a large series of bladder cancer patients (Catto et al, 2003). Here we will describe the modelling methods in further detail.

\section{PATIENTS AND MODEL PARAMETERS}

In total, 109 patients with primary TCC of the bladder, and clinical follow up of at least 5 years (median 6, range 5-16) were studied. Each tumour was graded using the WHO classification and staged according to the 1997 TNM classification (Sobin and Wittekind, 1997). There were 73 superficial (pTa and pT1) and 36 muscle invasive TCCs (pT2-4). Smoking data (total consumption and current status) were available for 107 patients; of whom 30 were nonsmokers, 34 were former (had stopped at least 1 year prior to presentation) and 43 were current smokers. There were no cases of suspected familial cancer syndromes. 'Relapse' was defined as the occurrence of a further tumour following initial treatment, proven either pathologically or radiologically. Local ethics committee approval was granted, and informed consent was obtained from all patients prior to commencement of the study. Further details are described elsewhere (Catto et al, 2003).

Immunohistochemistry was used to assess the 
mutational status in each tumour for the p53, hMLHl and hMSH2 genes, by standard methods described elsewhere (Cordon-Cardo et al, 1994; Thibodeau et al, 1996; Catto et al, 2003). Those tumours with absent or weak MMR staining ( $<30 \%$ of nuclei were positive) and excessive p53 staining ( $>20 \%$ positive nuclei) were scored as abnormal.

\section{ARTIFICIAL INTELLIGENCE MODELLING TECHNIQUES}

Two models were developed using both NFM and ANN as follows;

- A Classifier predicted the likelihood of a tumour relapse (yes or no).

- A Predictor predicted the timing of this relapse (months after surgery).

These two models were combined together in series; thus predicting both if and when a relapse would occur. To discover the value of the putative molecular biomarkers, the data were analysed twice in each model, as follows;

- Analysis (A) only the conventional clinicopathological data were studied.

- Analysis (B) both the conventional clinicopathological data and the additional 3 molecular putative biomarkers were studied.

The input variables used to train and test the NFM are shown in table 1 . The output variable from the models, was either the occurrence of relapse (Classifier) or the timing of relapse (Predictor).

\subsection{Neural Networks}

The Multi-Layer Perceptron (MLP) network is the most commonly used neural network for modelling. A MLP is a feedforward neural network typically consisting of an input layer, one or more hidden layers, and an output layer (Neelakanta, 1994). The power of the neural network approach is that it is generic in structure and it possesses the ability to 'learn' complex non-linear relationships with limited a priori knowledge about the process structure. The power of the MLP network has been demonstrated by a number of workers, and research has indicated that a feedforward network has the potential to approximate a continuous non-linear function with arbitrary accuracy. Figure 1 shows a typical architecture of a MLP network, which consists of a number of neurons arranged in three layers, an input layer a hidden layer, and an output layer. The number of neurons (processing elements) in the input layer and the output layer is determined by the nature of the problem, i.e., the dimension of the input and output variable of the process to be modelled, while the number of neurons in the hidden layer may vary and must be specified prior to the network training.

One of the commercially available packages for neural networks modelling is hosted by Matlab (Mathwork, 1999). The neural network toolbox provides a number of networks for modelling and control techniques for developing neural networks based application.

Table 1. Input variables for the modelling methods. Two analyses were performed, (A) and (B). For analysis (A) the inputs were the 6 conventional clinicopathological data. For analysis (B) there were 3 additional inputs, which were the results of experimental studies performed on p53, hMLH1 and hMSH2.

\begin{tabular}{|c|c|c|c|c|}
\hline & Input Variables & Scoring & & \\
\hline & Stage & $\mathrm{Ta}$ & $T 1$ & $\mathrm{~T} 2-4$ \\
\hline$\infty$ & Grade & 1 & 2 & 3 \\
\hline 4 & Age & In years & & \\
\hline$\underline{2}$ & Sex & & & \\
\hline$\stackrel{\Xi}{\Xi}$ & Smoking Exposure & Pack years & & \\
\hline & Previous Cancers (non-TCC) & $0=$ none & $1=1$ & $2=>2$ \\
\hline & $\mathrm{p} 53$ & $0=$ normal & $1=$ abnormal & \\
\hline $\bar{g}$ & hMLHl & $0=$ abnormal & $1=$ normal & \\
\hline$\infty$ & hMSH2 & $0=$ abnormal & $1=$ normal & \\
\hline
\end{tabular}




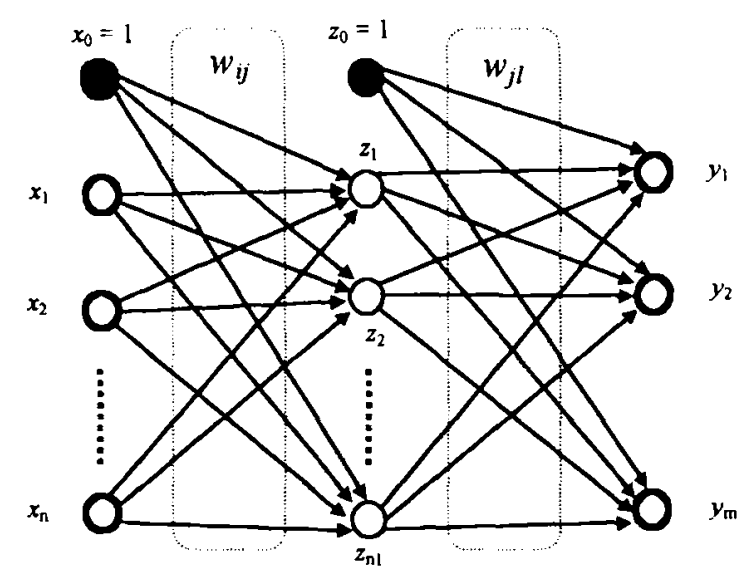

Input Layer Hidden Layer Output Layer

Fig 1. Architecture of a three layer Multi-Layer Perceptron (MLP)

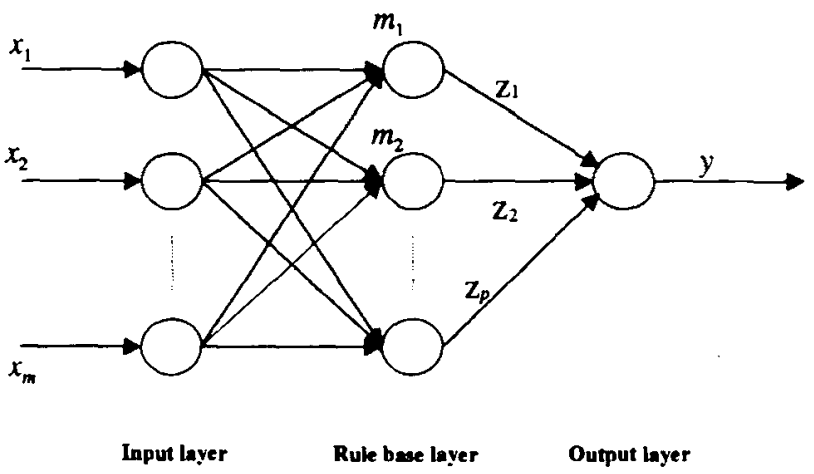

Fig 2. Basic structure of a neuro-fuzzy model.

\subsection{Neuro-Fuzzy Modelling}

The NFM analyses were performed via an extensive in-house suite of software developed in Matlab (Chen and Linkens, 2001). Figure 2 shows the architecture of a neuro-fuzzy system. A schematic diagram of this extensive suite is given in Figure 3. The modelling procedure involves a number of iterative loops subsequent to careful data preparation and initialisation of the starting model structure and parameters. These loops refine the models parameters, simplify its structure and component terms to the minimum complexity consistent with the model (i.e. parsimonious modelling), and validate the results

Most existing fuzzy modelling methods derive fuzzy models directly from raw data, our method provides a hierarchical clustering architecture based on a hybrid neural network for the fuzzy modelling procedure. Firstly, a fuzzy competitive neural network is exploited as a data pre-processor to extract a number of subclusters which can be viewed as an initial fuzzy model from raw data. This step is used to perform fuzzy classification with the objective of reducing the total number of training instances for the next stages. The number of clusters is determined by a classification phase which is based on the concept of maximum likelihood. Secondly, the problems of input selecting and fuzzy partition validating are dealt with separately on the basis of the initial fuzzy model. On one hand, a simplified fuzzy inference neural network is proposed to produce a fuzzy output for each input variable so that the importance of each input can be ranked and the significant input variables can be selected. On the other hand, we propose a modified fuzzy entropy measure as the fuzzy partition criterion based on the fuzzy c-means (FCM) clustering algorithm to decide the optimal number of fuzzy rules. Using this sub-cluster-based hierarchical clustering approach, the computation cost decreases greatly compared with data-based clustering because the effort to generate the sub-clusters via a competitive cluster network is relatively small even when it involves a large number of input variables. It is particularly important when generating the initial fuzzy model from high-dimensional data. Finally, we demonstrate this approach by applying it to different types of nonlinear system modelling.

\subsection{Linear Regression}

To compare the results obtain by NFM and NN, traditional statistical analysis is used (Linear regression). Previous authors have recomended to used linear regression as the traditional statistical method to compare to other modelling techniques (Sargent, 2001). The comparisons were performed using a $Z$ test. The significance of the relationships between the various clinicopathological and the immunohistochemical data were calculated using Fisher's exact test. A p value of $<0.05$ was taken to be significant.

\section{ENSEMBLE MODELLING}

\subsection{Biases and Variance}

It is necessary to point out why an ensembles of models performs better than a single model would do. Consider a dataset $\mathrm{D}$ with pairs of input-output data $\left(\mathrm{x}_{\mu}, \mathrm{y}_{\mu}\right), \mu=1, \ldots, \mathrm{N}$, where $x_{\mu} \in R^{d}$ and the $y_{\mu}$ are scalar values. We suppose an underlying functional relationship of the form $y=f(x)+\varepsilon$ where the additional error has zero mean and does not depend on $x$. The goal of regression is to find a close approximation $f(x)$ of the assumed deterministic 
function $f(x)$ with additional constrains:

- The modeling error (training error) should be as small as possible

- The generalization ability of the model $f(x)$ should be as high as possible, i.e. the error on unseen data sets, that were not used for model training, should also be small These two constrains mark a crucial problem in regression estimation, know as the bias-variance dilemma.

In order to reduce the training error, the complexity of the model should be increased, in general the number of free model parameters. This can be done, until the training error is zero, but a high outof-sample error is expected, because of the loss of generalization.

\subsection{Ensembles}

Averaging the output of several different models is called an ensemble of models, or simply an ensemble. The idea of averaging different models was developed in the neural network community in the beginning of the 90 's. It was pointed out by Krogh et al. (1995), that the generalization error of the ensemble is lower than the mean of the generalization error of the single ensemble members. To see this, we define the weighted ensemble average as:

$$
f(x)=\sum_{k=1}^{K} w_{k} f_{k}(x)
$$

where $f_{k}(x)$ denotes the k-individual model and the weights $\Sigma_{k} w_{k}=1$ sum to one.

The generalization error of the ensemble

$e(x)=(y(x)-f(x))^{2}$

can now be decomposed in the following manner:

$e(x)=\varepsilon(x)-a(x)$

with the two quantities

$$
e(x)=\sum_{k=1}^{K} w_{k}\left(y(x)-f_{k}(x)\right)^{2}
$$

Average error of the individual models

$$
a(x)=\sum_{k=1}^{K} w_{k}\left(f_{k}(x)-f(x)\right)^{2}
$$

Average ambiguity of the ensemble

Looking at this error decomposition, it can be concluded that:

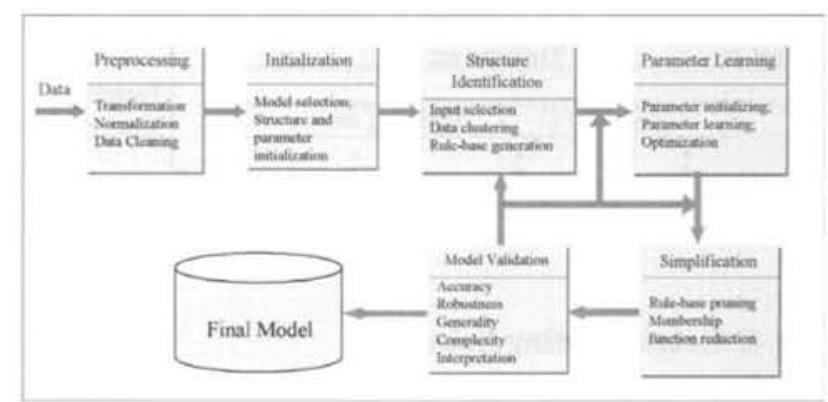

Fig 3. Schematic structure of the NFM suite of programs used to produce an optimal complexity model from the available data.

- The ensemble generalization error $e(x)$ is always smaller than the expected error of the individual models $\varepsilon(x)$

- An ensemble should consist of well trained but diverse models in order to increase the ensemble ambiguity

\subsection{Cross Validation}

In order to estimate the generalization error and to select models for the final ensemble, cross-validation scheme is used for model training (Hastie et al, 2001). The cross-validation is done in several training rounds on different training sets, because this increases the ambiguity of the ensemble and leads to better generalization (Krogh et al, 1995). Another advantage of this method is to get an unbiased estimator of the ensemble generalization error and at the same time training the ensemble on the whole data set. This is useful in situations, where only a few data points are available.

The whole procedure consists of the following steps:

- The data is divided in two sets

- Several models are trained on the first set

- The models are compared by evaluating the prediction errors on the unseen data of the test set

- The best performers are taken out and become ensemble members

- The data is divided again in a way that the new test set has minimal overlap with the former ones

- The procedure stops if the ensemble has the desired size

Figure 4 illustrates the data partition during the training of the cross-validation ensemble. 


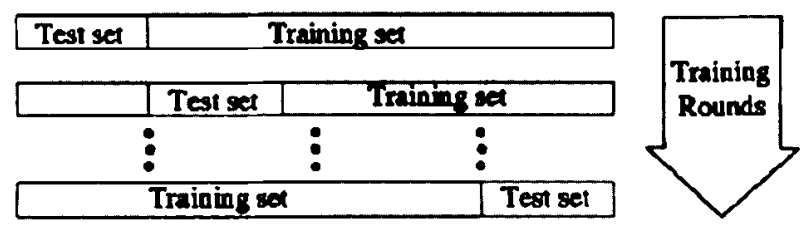

Fig 4. Cross training Scheme

Table 2. Risk of tumour relapse: results for the Classifier models. The table shows the results of the Classifiers' prediction of a tumour relapse (yes or no). In analysis (B), the additional putative molecular biomarkers are included, altering the performance of each model. (PPV=Positive predictive value, $N P V=$ Negative predictive value).

Sensitivity Specificity PPV NPV Accuracy

\begin{tabular}{rrrcccc}
\hline NFM A & 0.92 & 0.90 & 0.98 & 0.72 & 0.92 \\
& B & 0.90 & 0.80 & 0.92 & 0.74 & 0.88 \\
ANN A & 0.90 & 0.89 & 0.98 & 0.64 & 0.90 \\
& B & 0.94 & 0.96 & 0.99 & 0.84 & 0.95 \\
LR & A & 0.79 & 1.0 & 1.0 & 0.28 & 0.82 \\
& B & 0.87 & 1.0 & 1.0 & 0.61 & 0.89 \\
\hline
\end{tabular}

Table 3. Time to tumour relapse: results of the artificial intelligence Predictor models and logistic regression. The results of the $\mathrm{AI}$ models predictions and logistic regression probabilities of time to tumour relapse are shown, as root mean squares (difference between predicted and actual time of relapse). For ANN and NFM, after training and testing, the data are then analysed by the 'best fit' model.

Training Testing Best fit

\begin{tabular}{llccc} 
& & & & (ANN, NFM) \\
\hline NFM & A & 7.6 & 10.3 & 8.5 \\
& B & 7.2 & 7.6 & 7.3 \\
ANN & A & 9.1 & 12.3 & 8.8 \\
& B & 7.1 & 11.7 & 7.6 \\
LR & A & 13.1 & 12.7 & 12.6 \\
& B & 12.8 & 13.0 & 12.2 \\
\hline
\end{tabular}

\section{MODELLING RESULTS}

The results of the Classifier models generated using NFM and ANN are shown in table 2. The accuracies for NFM and ANN are 0.92 and 0.90 for analysis (A) and 0.88 and 0.95 for analysis (B), respectively. The results of the Predictor models are shown, in comparison with LR, in Table 3. In this table the difference between the actual and predicted time of relapse is shown as a root mean square value (RMS). In all categories the AI models perform better than LR, and NFM is more accurate than ANN. The significance of comparisons between these results is shown in Table 4. As can be seen, from Table 4(a), for all the data ANN and NFM are significantly superior to LR $(p<0.006)$. When NFM and ANN are directly compared, NFM is significantly better than ANN at predicting tumour relapse $(\mathrm{p}=0.01)$, in the testing phase, but for the best fit model the $p$ value falls just short of significance $(p=0.079)$. If analyses $(A)$ and $(B)$ are compared individually, Table 4(b), there is no difference between NFM and ANN for analysis (A) $(p=0.87)$, whilst for analysis (B), NFM is far superior to ANN $(\mathrm{p}<0.001)$. The predictions of all 3 methods are shown graphically, as scatter plots (Figure 5) and plotted by the Kaplan-Meier method in Figure 6 .

\section{DISCUSSION}

These results show that NFM can predict tumour behaviour with greater accuracy than both ANN and LR. Until the advent of AI, the best method of predicting tumour behaviour was logistic regression. However, the poor predictive accuracy of this method (69\%-73\% accurate (Burke et al, 1997)) and the fact that a general probability is not applicable to an individual patient, show the need for improvements. Using Figure 6, if LR were applied in clinical practice, those patients with late relapsing tumours (over 40 months) would have had their most intensive cystoscopic surveillance too early for their actual relapse. On an individual basis, those 2 patients with relapse after 60 months (both predicted to recur by 20 months) may have been falsely reassured and discharged.

Until recently, the accurate prediction of tumour behaviour has been mostly an academic exercise. However, recent discoveries in molecular medicine are revealing specific pathways of carcinogenesis, each with different treatment sensitivities. For example, tumours with high levels of microsatellite instability are resistant to DNA damaging chemotherapies (Drummond et al, 1996) and the p53 mutational status of a tumour can predict a successful response to 
chemotherapy (Cote, Chatterjee, 1995). Therefore, it has become more important to predict tumour behaviour. Previous workers have shown that $\mathrm{AI}$, in particular ANN, may provide suitable methods for this prediction. Reports have consistently shown that ANN is more accurate at predicting tumour behaviour than either LR (Burke et al, 1997; Porter et al, 2001; Potter et al, 1999; Tewari et al, 2001; Ziada, 2001) or clinicians (Qureshi et al, 2000). We have again confirmed that ANN provides a powerful and accurate predictive method. However, unlike these previous studies, we have been able to compare NFM with ANN. Our current study has shown that NFM produces a significantly more accurate prediction than ANN and LR (table 4).

Whilst the accuracy of NFM is important, it has many other benefits over ANN that promise to make it more acceptable to the clinical and scientific community. Unlike the 'black-box' approach of ANN, which makes interpretation of the model obtained from the data impossible, the NFM approach is transparent. The problem specific qualitative modelling representation can be easily translated into understandable medical terms. NFM uses the modelling abilities of fuzzy-logic to complete a profile

Table 4 (a). Statistical comparisons between artificial intelligence and logistic regression, for all data. The table shows the $P$ values of statistical comparisons ( $\mathrm{Z}$ test) between the three different methods. ANN and NFM are superior to $L R$ in training, but not testing, whilst NFM is superior to ANN in the testing phase. When all the data are analysed together, both $\mathrm{AI}$ methods are better than LR. This table compares the combined results of all the generated models. ${ }^{\star} \mathrm{P}<0.05$ is taken to be statistically significant.

\begin{tabular}{lccc} 
Comparison & Training & Testing & All data \\
\hline ANN v LR & $0.0006^{*}$ & 0.22 & $0.0004^{*}$ \\
NFM v LR & $0.009^{*}$ & 0.07 & $0.006^{*}$ \\
ANN v NFM & 0.15 & $0.01^{*}$ & 0.08 \\
\hline
\end{tabular}

Table 4 (b). Statistical comparisons between artificial intelligence and logistic regression, for individual analyses. As can be seen, both AI methods are superior to $L R$ for analysis $B$.

\begin{tabular}{lcc} 
Comparison & Analysis A & Analysis B \\
\hline NFM v ANN & 0.88 & $0.0001^{*}$ \\
ANN v LR & 0.06 & $0.004^{*}$ \\
NFM v LR & 0.19 & $0.01^{*}$ \\
\hline
\end{tabular}

for each variable. This produces a set of parallel rules, which are summated in series and interpreted to produce a quantitative output. An example is shown in Figure 7 . The top line, Rule 1, shows that a poorly differentiated (grade 3 ), superficially invasive tumour (stage $\mathrm{T} 1$ ), in a 70 year old male current smoker ( 30 cigarettes per day), with abnormal p53 staining, but otherwise normal variables, will have a short time to tumour relapse.

Once trained on a dataset the NFM suite is then able to reduce the inputs into those that have most influence. This is likely to assist clinicians in day-today clinical practice, where insufficient data may be present in a patients' casenotes. In Figure 8, the NFM has automatically reduced the 9 inputs to the 4 most effective ones. These can be interpreted to confirm that
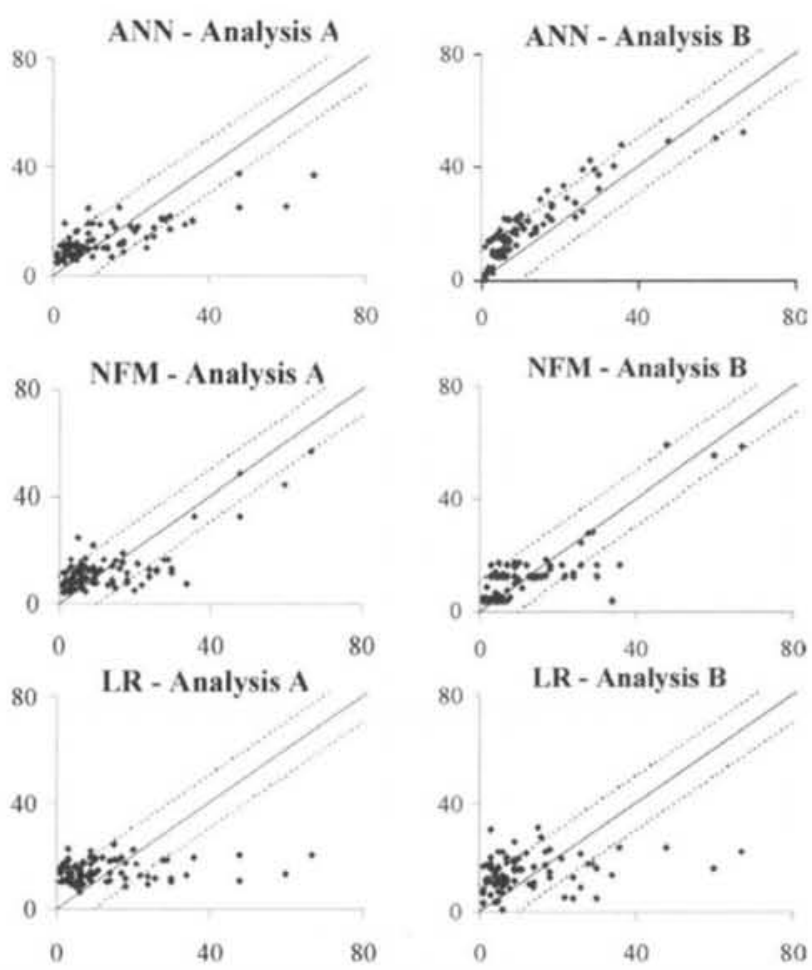

Fig 5. Scatter plots showing the actual and predicted times of tumour relapse. In all six graphs each point represents the actual time of tumour relapse ( $X$ axis; $0-80$ months after surgery) against the predicted time of tumour relapse ( $Y$ axis; $0-80$ months after surgery). The proximity of each point, to the line of perfect match (continuous line) indicates the accuracy of each plot. Dashed lines indicate the $\pm 10 \%$ range. ( $A N N=A r t i f i c i a l$ neural network, NFM=Neuro-fuzzy modelling and $L R=$ Logistic regression). 


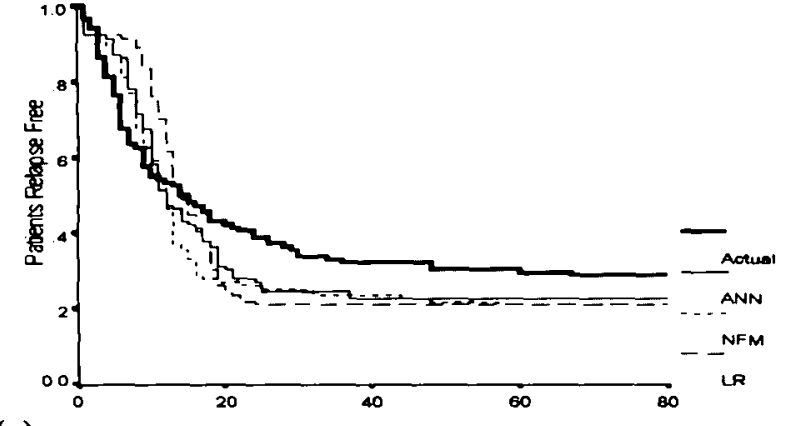

(a)

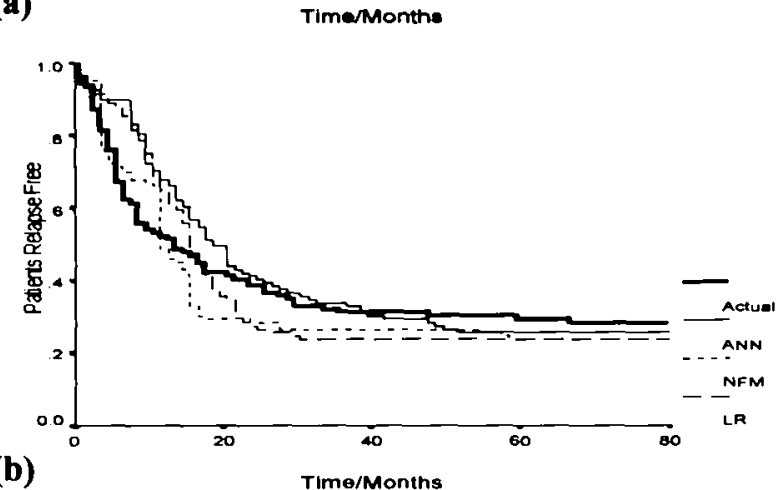

Fig 6. Kaplan-Meier plots of the actual, AI modelled and LR probabilities of tumour relapse. Figures $3 a$ and $b$ refer to analysis (A) (clinicopathological data only) and analysis (B), respectively.

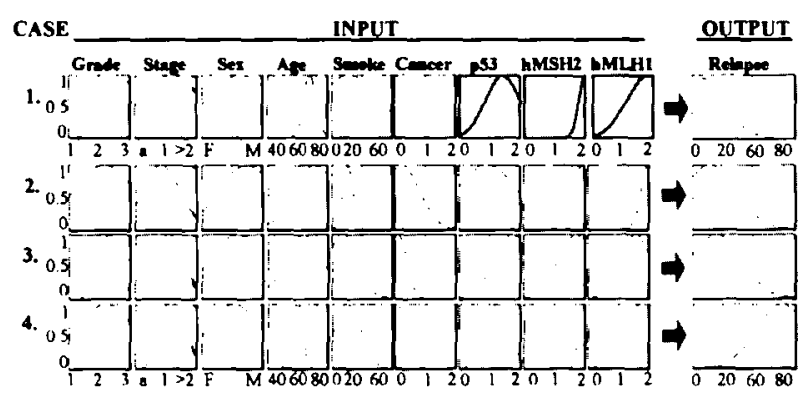

Fig 7. Neuro-Fuzzy Modelling output, after defuzzification. The above diagram represents the Modelling methods used by NFM. For each variable, quantitave points are joined in a qualitative manner using fuzzy-logic. The result is seen as a curve. When summated in series and interpreted, an output is produced, in this case; time to tumour relapse (months). See text for translation of case 1 . Case 1 and 4 have markedly different relapse times, probably due to the better differentiation of the tumour in case 4 , younger patient age, reduced smoking exposure and less abnormal p53 status.

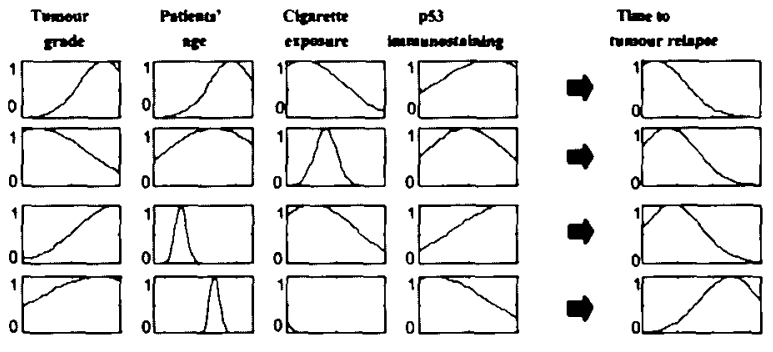

Fig 8. Neuro-Fuzzy Modelling using the most useful input variables. NFM has rationalised the 9 inputs from figure 4. to those that are most useful; Grade of tumour, Age of patient, Smoking exposure and p53 immunohistochemical status of the tumour. A linguistic interpretation of the first rule (top line) is that a grade 3 tumour, in $\mathbf{7 0}$ year old smoker, with abnormal p53 immunostaining is predicted to relapse at an early stage. Each of the models' rules can be interpreted linguistically to produce a translated chart (in figure 5 (b)).

these are clinically sensible inputs (tumour grade, patient age, smoking history and p53 expression). The rules can be linguistically interpreted as shown in Figure 9. The top line can be translated to show a welldifferentiated tumour, in a 65 years old medium smoker, has the shortest time to relapse. Interesting the NFM finds grade more useful than stage at predicting relapse, in agreement with traditional multivariate analysis.

The automatic selection of these inputs can easily be validated; for example, authors have used multivariate analysis of large TCC series to show that grade is more important than stage at predicting relapse (Millan-Rodriguez et al, 2001). This ability to prioritise the inputs will prove very useful in numerous clinical areas. For example, in this study we have shown that when compared indirectly, p53 is a more useful prognostic marker than hMLH1 and hMSH2. Therefore, NFM could be used relatively easily to screen the most promising molecular markers from large gene arrays (1000's of genes). Furthermore, because the fuzzy representation allows the predictive modelling rules to be understood, expertise can be incorporated into the selection of the inputs and manipulation of the models' rules. As a result nonsensible variables, which may be incidentally over represented in the training dataset, can be removed, or have their importance reduced. In addition, if there is medical knowledge available relevant to the NFM rule set, this can be added to the model easily. This will then enable the model to generalise and be more robust than other AI approaches. 


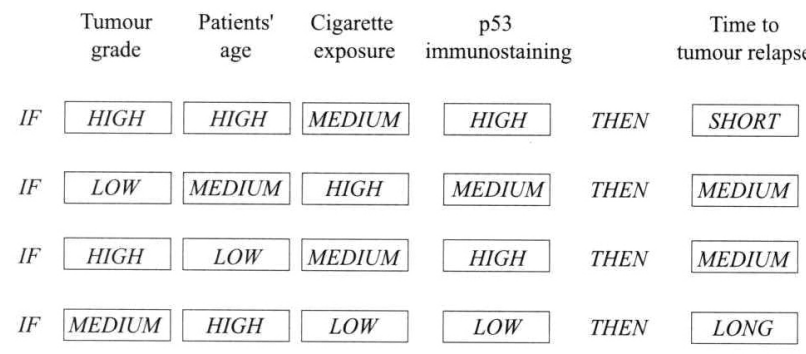

Fig 9. Neuro-fuzzy output using a linguistsic representation. In each box a word has replaced the output graph seen in 5 (a). It should be emphasised that these are fuzzy words and make sense within that models' rule, not necessarily in isolation.

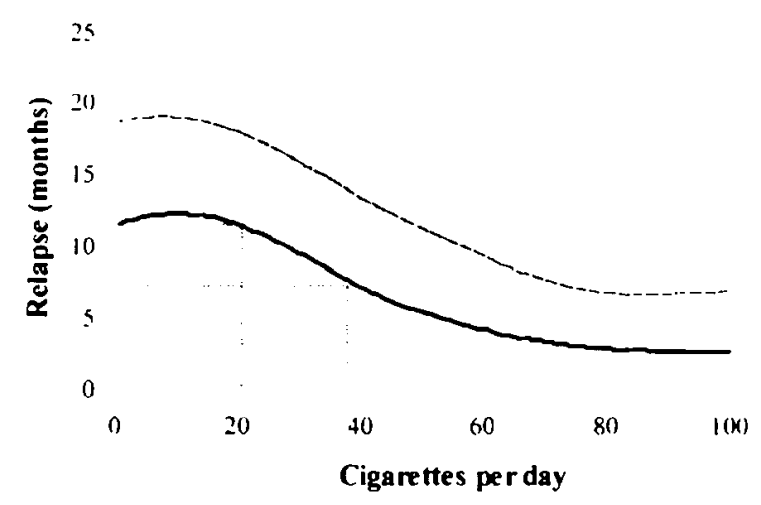

Fig 10. A graph showing the time of bladder cancer relapse against the number of cigarettes smoked per day for an individual patient. The curve shows the predicted time of relapse (months after surgery) for a 70 year old male patient with a poorly differentiated superficially invasive bladder tumour (G3 pT1) with normal molecular markers. The $10 \%$ confidence interval is shown as the dashed curve. As the two plotted points show, if the patient reduces smoking from 40 to 20 cigarettes per day, the time to relapse will increase from 6 to 11 months.

The modelling abilities of NFM have further benefits. It is possible to hypothetically change a single variable, whilst keeping the others constant, and produce new exploratory predictions. This allows both hypothesis testing and individual risk assessment to be performed. In Figure 10, NFM has modelled an individual patient's tumour relapse (in months) against number of cigarettes they smoke per day. The other inputs were fixed to represent a typical patient; a 70 year old man with a poorly differentiated, superficially invasive TCC (G3pTI). From the resultant model, it can be seen that if smoking were reduced from 40 per day to 20 per day, the time to relapse would increase from 6 to 12 months.

\section{CONCLUSIONS}

It has been demonstrated that NFM can successfully predict the occurrence and timing of tumour relapse, with similar or greater accuracy than linear regression. NFM modelling can benefit from many features as follows: its transparency features allow the ability to incorporate expertise, the superior performance with sparse data, the ability to select the most useful input criteria (parsimony) and to allow predictions of outcome that result from changes in the value of individual inputs. These features suggest that NFM could be used as a valuable and versatile tool to address numerous clinical situations. Whilst the predictions have been performed using bladder cancer data, these methods are transferable to all other human malignancies.

\section{REFERENCES}

1. Quinn M. Cancer trends in England and Wales 1950-1999. SMPs No. 66 ed: The Stationary Office, 2000.

2. Hall RR, Parmar MK, Richards AB, Smith PH. Proposal for changes in cystoscopic follow up of patients with bladder cancer and adjuvant intravesical chemotherapy. $\mathrm{Br}$ Med J 1994;308(6923):257-60.

3. Stein JP, Lieskovsky G, Cote RJ, al. e. Radical cystectomy in the treatment of invasive bladder cancer; long term results in 1054 patients. J Clinical Oncology 2001;19:666-75

4. Raghaven D, Quinn D, Skinner DG, Stein JP. Surgery and adjunctive chemotherapy for invasive bladder cancer. Surg Oncol 2002;11:55-63.

5. Sobin LH, Wittekind $\mathrm{CH}$. TNM classification of malignant tumours. 5th ed. New York (NY): John Wiley and sons, 1997.

6. Parmar MKB, Freedman L.S, Hargreave TB, Tolley DA. Prognostic factors for recurrence and follow up policies in the treatment of superficial bladder cancer: interim report from the British Medical Research Council subgroup on superficial bladder cancer (Urological cancer working party). J Urol 1989;142:284-8.

7. Hollstein T, Sidransky D, Vogelstein B, Harris CC. p53 mutations in human cancers. Science 1991;253:49. 
8. Esrig D, Elmajian D, Groshen $S$, et al. Accumulation of nuclear p53 and tumour progression in bladder cancer. $\mathrm{N}$ Engl $\mathrm{J}$ Med 1994;331:1259.

9. Aaltonen LA, Peltomaki P, Leach FS, et al. Clues to the pathogenesis of familial colorectal cancer. Science 1993;260(5109):812-6.

10. Liu B, Nicolaides NC, Markowitz S, et al. Mismatch repair gene defects in sporadic colorectal cancers with microsatellite instability. Nat Genet $1995 ; 9(1): 48-55$.

11. Drummond JT, Anthoney A, Brown R, Modrich P. Cisplatin and adriamysin resistance are associated with MutLA and mismatch repair deficiency in an ovarian tumour cell line. J. Biol. Chem. 1996;271(33):19645-19648.

12. Gryfe R, Kim H, Hsieh ET, et al. Tumor microsatellite instability and clinical outcome in young patients with colorectal cancer. $\mathrm{N}$ Engl $\mathbf{J}$ Med 2000;342(2):69-77.

13. Burke HB, Goodman PH, Rosen DB, et al. Artificial neural networks improve the accuracy of cancer survival prediction. Cancer 1997;79:857-62.

14. Baxt WG. Use of an artificial neural network for data analysis in clinical decision-making: the diagnosis of acute coronary occlusion. Neural Computation 1989;2:480-89.

15. Cross SS, Harrison RF, Kennedy RL. Introduction to neural networks. Lancet 1995;346:1075-79.

16. Baxt WG. Application of artificial neural networks to clinical medicine. Lancet 1995;346:1135-8.

17. Qureshi KN, Naguib RNG, Hamdy FC, Neal DE, Mellon JK. Neural network analysis of clinicopathological and molecular markers in bladder cancer. J Urol 2000;163:630-633.

18. Nie J, Linkens DA. Fuzzy-neural control. London: Prentice Hall, 1995.

19. Thibodeau SN, French AJ, Roche PC, et al. Altered expression of $\mathrm{hMSH} 2$ and $\mathrm{hMLH} 1$ in tumors with microsatellite instability and genetic alterations in mismatch repair genes. Cancer Res 1996;56(21):4836-40.

20. Cordon-Cardo C, Dalbagni G, Saez GT, et al. p53 mutations in human bladder cancer: genotypic versis phenotypic patterns. Int $\mathrm{J}$ Cancer 1994;56:347-53.
21. Chen M, Linkens DA. A systematic neurofuzzy modelling framework with application to material property prediction. IEEE Trans SMC. Part B: Cybernetics. 2001;31(5):781-790.

22. Sargent DJ. Comparison of artificial neural networks with other statistical approaches. Cancer 2001;91:1636-42.

23. Cote RJ, Chatterjee SJ. Molecular determinants of outcome in bladder cancer. Cancer J Sci Am 1999;5:1.

24. Porter C, O'Donnel C, Crawford DE, et al. Artificial neural network model to predict biochemical failure after radical prostatectomy. Mol Urol 2001;5(4):159-62.

25. Potter SR, Miller C, Mangold LA, et al. Genetically engineered neural networks for predicting prostate cancer progression after radical prostatectomy. Urology 1999;54:791-5.

26. Tewari A, Issa M, El-Galley R, et al. Genetic adaptive neural network to predict biochemical failure after radical prostatectomy: a multi institutional study. Mol Urol 2001;5(4):163-9.

27. Ziada AM, Lisle TC, Snow PB, Levine RF, Miller $G$, Crawford DE. Impact of different variables on the outcome of patients with clinically confined prostate cancer. Cancer 2001;91:1653-60.

28. Millan-Rodriguez F, Chechile-Toniolo G, SalvadorBayarri J, Palou J, Vicente-Rodriguez J. Multivariate analysis of the prognostic factors of primary superficial bladder cancer. J Urol 2000;163(1):73-8.

29. Catto, J.W.F., Linkens, D.A., Abbod, M.F., Chen, M., Burton, J.L., Feeley, K.M., and Hamdy, F.C. Artificial Intelligence in Predicting Bladder Cancer Outcome: A Comparison of Neuro-Fuzzy Modeling and Artificial Neural Networks. Clinical Cancer Research, 2003:9:4172-4177.

30. Matlhworks, Matlab Version 5.3, http://www.mathworks.com, 1999.

31. Neelakanta, P.S., Neural Network Modelling: Statistical Mechanics and Cybernetic Perspectives, Boca Raton, Florida, London : CRC Press, 1994.

32. Hastie, Tibshirani, Friedman: The Elements of Statistical Learning, Springer, 2001.

33. Krogh, Vedelsby: Neural Network Ensembles, Cross Validation and Active Learning, Advances in Neural Information Processing Systems 7, MIT Press, 1995. 